\title{
Patient-reported outcomes in patients with cystic fibrosis with a G551D mutation on ivacaftor treatment: results from a cross- sectional study
}

Scott C. Bell ${ }^{1 *}$, Jochen G. Mainz ${ }^{2,3}$, Gordon MacGregor ${ }^{4}$, Susan Madge ${ }^{5}$, Julie Macey ${ }^{6}$, Moshe Fridman, Ellison D. Suthoff ${ }^{8}$, Siva Narayanan ${ }^{9}$ and Nils Kinnman ${ }^{10}$

\begin{abstract}
Background: Clinical studies demonstrate that ivacaftor (IVA) improves health-related quality of life (HRQLL) in patients aged $\geq 6$ years with cystic fibrosis (CF). The real-world impact of IVA and standard of care (SOC) in groups of patients with G551D and F508del mutations, respectively, was assessed using a survey comprising disease-specific and generic HRQoL measures.

Methods: Patients with CF aged $\geq 12$ years, or aged 6-11 years with caregiver support, with either (1) a G551D mutation and receiving IVA (G551D/VA) for $\geq 3$ months, or (2) homozygous for F508del and receiving SOC before lumacaftor/IVA availability (F508del/SOC), were eligible to participate in a cross-sectional survey. Demographic and clinical characteristics, and HRQoL measures were compared between patient groups, and multiple regression analyses were conducted.

Results: After differences in patient demographic and clinical characteristics were controlled for, significantly better scores were observed in the G551D/VA group than in the F508del/SOC group on multiple domains of the validated Cystic Fibrosis Questionnaire-Revised and the EuroQol 5-dimensions 5-level questionnaire.

Conclusions: G551D/IVA patients reported better HRQoL than F508del/SOC patients on generic and disease-specific measures in a real-world setting.
\end{abstract}

Keywords: Patient-reported outcomes, Work productivity, Ivacaftor, Cystic fibrosis, G551D-CFTR

\section{Background}

Cystic fibrosis (CF) is a chronic, progressive, lifeshortening disease that affects the ability of patients to function in many areas of daily living $[1,2]$. There is growing evidence that patient-reported outcomes (PROs) can serve as valuable indicators of the benefit of treatment and its impact on health-related quality of life (HRQoL), as they represent direct measures of how a patient feels and functions $[3,4]$. Evaluation of such measures is becoming increasingly important for patients with chronic disease, such as $\mathrm{CF}$, which necessitates complex

\footnotetext{
* Correspondence: scott.bell@qimrberghofer.edu.au

'Department of Thoracic Medicine, The Prince Charles Hospital and QIMR

Berghofer Medical Research Institute, Brisbane, QLD, Australia

Full list of author information is available at the end of the article
}

management regimens and imposes a considerable burden on patients and their caregivers $[1,5,6]$.

The Cystic Fibrosis Questionnaire-Revised (CFQ-R) is a disease-specific, validated tool that encompasses general domains of HRQoL as well as domains specific to CF $[7,8]$. The EuroQol 5-dimensions 5-level questionnaire (EQ-5D-5L) is a generic measure that provides a single index value of health status and permits comparisons between treatments across different medical conditions $[4,9,10]$. The EQ-5D-5L is a widely accepted measure for assessing utilities, which is recommended by the National Institute for Health and Care Excellence in the United Kingdom (UK), and is a standardized assessment that can be used across a variety of diseases [11]. Other PROs, such as the Work Productivity and Activity Impairment Questionnaire (WPAI) [12, 13],

(c) The Author(s). 2019 Open Access This article is distributed under the terms of the Creative Commons Attribution 4.0 International License (http://creativecommons.org/licenses/by/4.0/), which permits unrestricted use, distribution, and reproduction in any medium, provided you give appropriate credit to the original author(s) and the source, provide a link to the Creative Commons license, and indicate if changes were made. The Creative Commons Public Domain Dedication waiver (http://creativecommons.org/publicdomain/zero/1.0/) applies to the data made available in this article, unless otherwise stated. 
take productivity losses into consideration, which may provide additional context for the impact of treatment on patients and their caregivers in the real world.

The treatment benefit of ivacaftor (IVA) in patients with CF with a G551D mutation in the cystic fibrosis transmembrane conductance regulator (CFTR) gene has been established in Phase 3 studies [14-16]. Improvements in lung function, body weight, and HRQoL, as measured by the CFQ-R Respiratory Symptoms domain, were observed through 48 weeks $[14,15]$; these benefits were sustained for nearly 3 years in an open-label extension study [16]. An additional analysis using Phase 3 data further demonstrated broad HRQoL benefits of IVA across multiple domains of the CFQ-R [17]. However, the majority of HRQoL data with IVA have been derived from clinical trials [14-17], whereas HRQoL data from real-world studies are limited [18]. We therefore sought to expand on these studies by determining the impact of IVA on HRQoL using the CFQ-R and EQ-5D-5L, and work productivity using the WPAI. We also sought to compare outcomes for patients with the G551D-CFTR mutation on $\geq 1$ allele who were receiving treatment with IVA (G551D/ IVA group) in the real-world setting with those for patients homozygous for the F508del-CFTR mutation who were receiving standard of care (SOC) (F508del/SOC group) treatment prior to availability of lumacaftor (LUM)/IVA.

\section{Methods}

Patients with CF who were $\geq 12$ years of age and caregivers of patients aged 6-11 years were recruited to participate in this cross-sectional, observational study in 5 countries (France, the UK, Germany, Australia, and Ireland). The final study report date was October 2, 2014. Patients were screened by the site coordinators at the time of clinic visits to confirm study eligibility and to collect demographic and clinical characteristics. Patients who were eligible for the study were diagnosed with CF, had $\geq 1$ G551D mutation, and were receiving IVA with treatment exposure for $\geq 3$ months, or were homozygous for the F508del mutation and receiving SOC (not exposed to or receiving IVA and prior to the availability of LUM/IVA). Patients were excluded from the study if they were participating in an interventional clinical trial, experiencing a pulmonary exacerbation at their clinic visit, or unable or unwilling to provide consent (adults/caregivers) or assent (minors). A study screening form, completed by clinic staff, reported demographic and clinical patient data, such as percent predicted forced expiratory volume in $1 \mathrm{~s}\left(\mathrm{ppFEV}_{1}\right)$, height, weight, CFTR mutations, select key comorbidities (i.e. presence of anxiety, attention deficit hyperactivity disorder, depression, diabetes, cancer, infertility, end-stage liver disease, pulmonary hypertension, osteoporosis, pancreatic insufficiency, pancreatitis, chronic rhinosinusitis/ nasal polyps, and rectal prolapse), and current CF treatment including IVA.

Patients or their caregivers who qualified and consented to participate in the study completed a one-time survey at the time of their clinic visit comprising the CFQ-R $[7,8]$, EQ-5D-5 L $[9,10]$, and WPAI $[12,13]$. Three distinct agespecific versions of the survey were used for patients aged 6-11 years, $12-13$ years, and $\geq 14$ years. The survey was completed by the patients themselves with the following exceptions: patients aged 6-11 years (caregivers completed the survey) and patients aged $\geq 12$ years (completed by the patient, with some assistance from the caregiver, as appropriate).

The CFQ-R measures HRQoL in general domains of Vitality, Health Perceptions, Physical Functioning, Emotional Functioning, Social Functioning, and Role Functioning, and CF-specific domains of Body Image, Eating, Treatment Burden, and Respiratory and Digestive Symptoms $[7,8]$. The EQ-5D-5L has a descriptive system that measures general health status in 5 dimensions: Mobility, Self-Care, Usual Activities, Pain/Discomfort, and Anxiety/ Depression, and a visual analog scale (VAS) that records an individual's self-rated health on a scale of 0-100 [10]. Both the CFQ-R and EQ-5D-5L have been validated in $\mathrm{CF}[7,8,10]$. The WPAI is designed to measure the effect of general health and symptom severity on work/school productivity and regular activities during the past 7 days; it has been validated in respiratory disease, but not in CF [12, 13].

Demographic and clinical characteristics of the G551D/ IVA and F508del/SOC patient groups were compared, with $t$-tests for numeric variables and $\chi^{2}$ tests for categorical variables. Multivariate regression analyses were conducted for outcomes on the CFQ-R, EQ-5D-5L, and WPAI to compare G551D/IVA and F508del/SOC groups, adjusting for differences observed between groups in sex, $\mathrm{ppFEV}_{1}$, and number of comorbidities. Multiplicity was not controlled for. Least squares (LS) estimated means were presented as adjusted mean values. $P$ values $<0.05$ were considered statistically significant in all analyses.

Central ethics approval of the study protocol was obtained in the UK and France, while institution-specific ethics approval was obtained at each of the participating institutions from Germany, Australia, and Ireland.

\section{Results}

Demographic and clinical characteristics of 209 survey respondents (72 in the G551D/IVA group and 137 in the F508del/SOC group) at study entry are shown in Table 1. In the G551D/IVA group, of the 72 surveys, 60 were completed by patients aged $\geq 12$ years and 12 by caregivers for children aged 6-11 years. In the F508del/ SOC group, of the 137 surveys, 116 were completed by patients aged $\geq 12$ years and 21 by caregivers for 
Table 1 Demographic and clinical characteristics

\begin{tabular}{|c|c|c|c|c|}
\hline Characteristic & $\begin{array}{l}\text { Overall } \\
(N=209)\end{array}$ & $\begin{array}{l}\text { G551D/IVA } \\
\text { patient group } \\
(n=72)\end{array}$ & $\begin{array}{l}\text { F508del/SOC } \\
\text { patient group } \\
(n=137)\end{array}$ & $P$ value \\
\hline Age, mean (SD), years & $24.3(12.1)$ & $23.9(13.9)$ & $24.6(11.1)$ & 0.70 \\
\hline Range & $6-62$ & $6-62$ & $6-52$ & \\
\hline Missing, $n$ & 6 & 3 & 3 & \\
\hline \multicolumn{5}{|l|}{ Age group (years), $n(\%)$} \\
\hline $6-11$ & $33(15.8)$ & $12(16.7)$ & $21(15.3)$ & \\
\hline $12-17$ & $39(18.7)$ & $18(25.0)$ & $21(15.3)$ & 0.19 \\
\hline$\geq 18$ & $137(65.6)$ & $42(58.3)$ & $95(69.3)$ & \\
\hline \multicolumn{5}{|l|}{ Sex, $n(\%)^{b}$} \\
\hline Male & $108(56.0)$ & $27(39.7)$ & $81(64.8)$ & $<0.01$ \\
\hline Female & $85(44.0)$ & $41(60.3)$ & $44(35.2)$ & \\
\hline Missing, $n$ & 16 & 3 & 12 & \\
\hline $\mathrm{ppFEV}_{1}(\%)$, mean $(\mathrm{SD})$ & $73.9^{c}(28.0)$ & $79.8(25.6)$ & $70.7^{d}(28.8)$ & $<0.05$ \\
\hline $\mathrm{ppFEV}_{1}<70, n(\%)$ & $85(40.7)^{e}$ & $24(33.3)$ & $61(44.5)^{\mathrm{e}}$ & 0.12 \\
\hline \multicolumn{5}{|l|}{$\mathrm{ppFEV}_{1}(\%), n$; mean (SD) } \\
\hline$<40$ & $32 ; 29.3(6.6)$ & $6 ; 28.8(4.5)$ & $26 ; 29.3(7.0)$ & 0.87 \\
\hline $40-69$ & $51 ; 54.5(8.7)$ & $18 ; 57.4(7.9)$ & $33 ; 52.9(8.7)$ & 0.07 \\
\hline $70-89$ & $50 ; 79.2(6.0)$ & $16 ; 78.6(6.6)$ & $33 ; 79.5(5.7)$ & 0.60 \\
\hline$\geq 90$ & $74 ; 103.0(10.5)$ & $31 ; 103.4(9.7)$ & $43 ; 102.6(11.1)$ & 0.74 \\
\hline Number of comorbidities, mean (SD) & $1.9^{f}(1.2)$ & $1.5^{9}(1.1)$ & $2.0(1.2)$ & $<0.01$ \\
\hline Patients with $\geq 1$ comorbidity, $n$ (\%) & $192(92.3)$ & $60(84.5)$ & $132(96.4)$ & $<0.01$ \\
\hline \multicolumn{5}{|l|}{ History of lung transplant, $n$ (\%) } \\
\hline Yes & $3^{f}(1.4)$ & $0^{9}(0.0)$ & $3(2.2)$ & 0.55 \\
\hline No & $205^{f}(98.6)$ & $71^{9}(100.0)$ & $134(97.8)$ & \\
\hline \multicolumn{5}{|l|}{ Waiting for lung transplant, $n$ (\%) } \\
\hline Yes & $3^{f}(1.4)$ & $0^{9}(0.0)$ & $3(2.2)$ & 0.55 \\
\hline No & $205^{f}(98.6)$ & $71^{9}(100.0)$ & $134(97.8)$ & \\
\hline \multicolumn{5}{|l|}{ BMl } \\
\hline Aged $\geq 19$ years, $n$; mean $(S D), \mathrm{kg} / \mathrm{m}^{2}$ & $126 ; 21.6(3.3)$ & $36 ; 22.2(3.2)$ & $90 ; 21.3(3.3)$ & 0.16 \\
\hline Aged $<19$ years, $n$; mean (SD) $z$-score & $59 ; 0.001(0.87)$ & $25 ; 0.004(0.82)$ & $34 ;-0.0009(0.92)$ & 0.98 \\
\hline
\end{tabular}

$B M I$ Body mass index, IVA Ivacaftor, ppFEV ${ }_{1}$ Percent predicted forced expiratory volume in $1 \mathrm{~s}$, SD Standard deviation, SOC Standard of care

${ }^{a} P$ values presented are for comparison of the G551D/IVA group with the F508del/SOC group

${ }^{b}$ Percentages are derived from the available sample

${ }^{c} n=207$

${ }^{d} n=135$

${ }^{\mathrm{e}}$ Baseline data was missing for 2 patients

${ }^{\mathrm{f}} n=208$

$\mathrm{g}_{n=71}$

children aged 6-11 years. Overall, the mean (standard deviation [SD]) patient age was 24.3 (12.1) years and $56 \%$ of patients were male (Table 1). Some differences were observed between groups. Age was similar between the two groups but the G551D/IVA group had a significantly higher proportion of females than the F508del/SOC group $(60.3 \%$ vs. $35.2 \%$, respectively; $P<0.01)$. The G551D/IVA group also had a significantly higher mean $\mathrm{ppFEV}_{1}$ than the $\mathrm{F}$ 08del/SOC group (79.8 vs. 70.7 percentage points, respectively;
$P<0.05)$, significantly fewer patients with pancreatic insufficiency $(80.3 \%$ vs. 92.0\%; $P<0.05)$, significantly fewer mean number of comorbidities (1.5 vs. 2.0; $P<0.01$ ), and significantly lower proportion of patients with $\geq 1$ comorbidity $(84.5 \%$ vs. $96.4 \% ; P<0.01)$ (Table 1). Individual comorbidities for which significant differences were observed between G551D/IVA and $F 508 \mathrm{del} / \mathrm{SOC}$ groups included pancreatitis $(2.8 \%$ vs. $0.0 \% ; P<0.05)$ and nasal polyp formation $(8.5 \%$ vs. $19.0 \% ; P<0.05$ ). Body mass index (BMI) for those 
aged $\geq 19$ years was similar between the groups, as was BMI $\mathrm{z}$-score for those aged $<19$ years.

The mean (SD) duration of IVA exposure was 21.8 (15.1) months. Patient recruitment and duration of IVA exposure varied by country, as shown in Table 2 .

Using an analysis of covariance model controlled for differences in $\mathrm{ppFEV}_{1}$, sex, and number of comorbidities at the time of their clinic visit, patients in the G551D/IVA group had significantly higher LS mean CFQ-R scores than patients in the F508del/SOC group in the following scales: Respiratory Symptoms (75.4 vs. $62.5 ; P<0.0001)$, Digestive Symptoms (85.5 vs. 78.0; $P<0.05)$, Eating (91.1 vs. $84.2 ; P<0.05)$, Treatment Burden (65.3 vs. 54.8; $P<0.01$ ), and Physical Functioning (74.6 vs. 66.6; $P<0.05$ ) (Fig. 1). Patients in the G551D/IVA group also had significantly higher LS mean CFQ-R scores than patients in the F508del/SOC group in the 6-11-year and the $\geq 14$-year versions of the Weight (80.7 vs. 64.2; $P<0.01$ ), Health Perceptions (67.6 vs. 58.6; $P<0.01$ ), and Vitality (63.5 vs. 55.9; $P<0.05)$ (Fig. 1$)$ domains. For the EQ-5D-5L, significantly better LS mean index scores and VAS scores were also observed in the G551D/IVA group than in the F508del/SOC group after controlling for differences in $\mathrm{ppFEV}_{1}$, sex, and number of comorbidities (Table 3). Within individual domains of the EQ-5D-5L, significantly better scores of Mobility, Usual Activities, Pain/Discomfort, and Anxiety/Depression were observed for the G551D/IVA group, whereas Self-Care was not

Table 2 Patient cohorts by country with duration of IVA exposure

\begin{tabular}{|c|c|c|c|}
\hline Country & Overall & $\begin{array}{l}\text { G551D/IVA } \\
\text { patient group }\end{array}$ & $\begin{array}{l}\text { F508del/SOC } \\
\text { patient group }\end{array}$ \\
\hline All countries, $N$ & 209 & $72^{a}$ & 137 \\
\hline $\begin{array}{l}\text { IVA exposure, } \\
\text { mean (SD), months }\end{array}$ & NA & $21.8(15.1)$ & NA \\
\hline France, $n(\%)$ & $61(29.2)$ & $10(13.9)$ & $51(37.2)$ \\
\hline $\begin{array}{l}\text { IVA exposure, } \\
\text { mean (SD), months }\end{array}$ & NA & $15.9(8.4)$ & NA \\
\hline United Kingdom, n (\%) & $54(25.8)$ & $33(45.8)$ & $21(15.3)$ \\
\hline $\begin{array}{l}\text { IVA exposure, } \\
\text { mean (SD), months }\end{array}$ & NA & $18.1^{\mathrm{b}}(11.4)$ & NA \\
\hline Germany, n (\%) & $47(22.5)$ & $14(19.4)$ & $33(24.1)$ \\
\hline $\begin{array}{l}\text { IVA exposure, } \\
\text { mean (SD), months }\end{array}$ & NA & $19.6(9.6)$ & NA \\
\hline Australia, n (\%) & $38(18.2)$ & $12(16.7)$ & $26(19.0)$ \\
\hline $\begin{array}{l}\text { IVA exposure, } \\
\text { mean (SD), months }\end{array}$ & NA & $35.6(24.1)$ & NA \\
\hline Ireland, $n$ (\%) & $9(4.3)$ & $3(4.2)$ & $6(4.4)$ \\
\hline $\begin{array}{l}\text { IVA exposure, } \\
\text { mean (SD), months }\end{array}$ & NA & $30.0(0.0)$ & NA \\
\hline
\end{tabular}

IVA Ivacaftor, NA Not applicable, SD Standard deviation, SOC Standard of care ${ }^{a}$ Duration on ivacaftor exposure was missing for 6 patients $\mathrm{b}_{n}=27$ significantly different between the groups (Fig. 2). In adjusted analyses of the WPAI, LS mean scores for School Productivity Loss and Daily Activity Impairment were numerically lower in the G551D/IVA group than in the $F 508 \mathrm{del} / \mathrm{SOC}$ group, although differences between groups did not reach statistical significance (Table 3).

\section{Discussion}

This cross-sectional observational study demonstrated that treatment with IVA in patients with CF and a G551D mutation was associated with multiple HRQoL benefits on disease-specific and generic and validated PRO measures compared with patients with CF who were homozygous for F508del and receiving SOC. This study was designed to evaluate outcomes following IVA exposure for a longer period of time than the usual follow-up period of randomized controlled trials, with the mean duration of IVA exposure being 21.8 months. Better outcomes on the CFQ- $\mathrm{R}$ in multiple domains and on the EQ-5D-5L using both index and VAS scores were observed in the G551D/IVA group. These findings lend additional real-world support to the HRQoL benefit of IVA previously described in clinical trials [14-17].

In recent years, there has been mounting interest from regulatory authorities as well as funding authorities to evaluate HRQoL, efficacy, and safety in real-world settings, necessitating continued demonstration of health outcomes. In particular, measures that can generate a single index value to allow for comparisons across a disease state that contribute to cost analysis are needed [4]. Data on EQ-5D-5L scores are instrumental in health technology assessments (HTA) in several countries around the world [11]. In this analysis, significant differences between the G551D/IVA and F508del/SOC groups were observed on the EQ-5D-5L using both the index score and VAS. Although a minimum clinically important difference has not yet been established in $\mathrm{CF}$, in chronic obstructive pulmonary disease, the mean estimates of the minimum clinically important difference for the utility index and EuroQol-VAS have been estimated at 0.051 (range 0.037-0.063) and 6.9 (range 6.58.0), respectively [19]. This is in line with the difference of 0.09 observed in this study. It should be noted that children with CF and their parents tend to develop coping mechanisms early in life and often establish a new normal' in their minds. This is sometimes reflected in high EQ-5D-5L scores, as seen in this study, and can make it difficult to identify differences in treatment effects using the EQ-5D-5L. Despite this, the statistically significant difference in the tool measures shown here mark a notable finding in this area.

With respect to the subscales on the EQ-5D-5L, we saw better scores for Mobility, Usual Activities, Pain/ Discomfort, and Anxiety/Depression in the G551D/IVA 


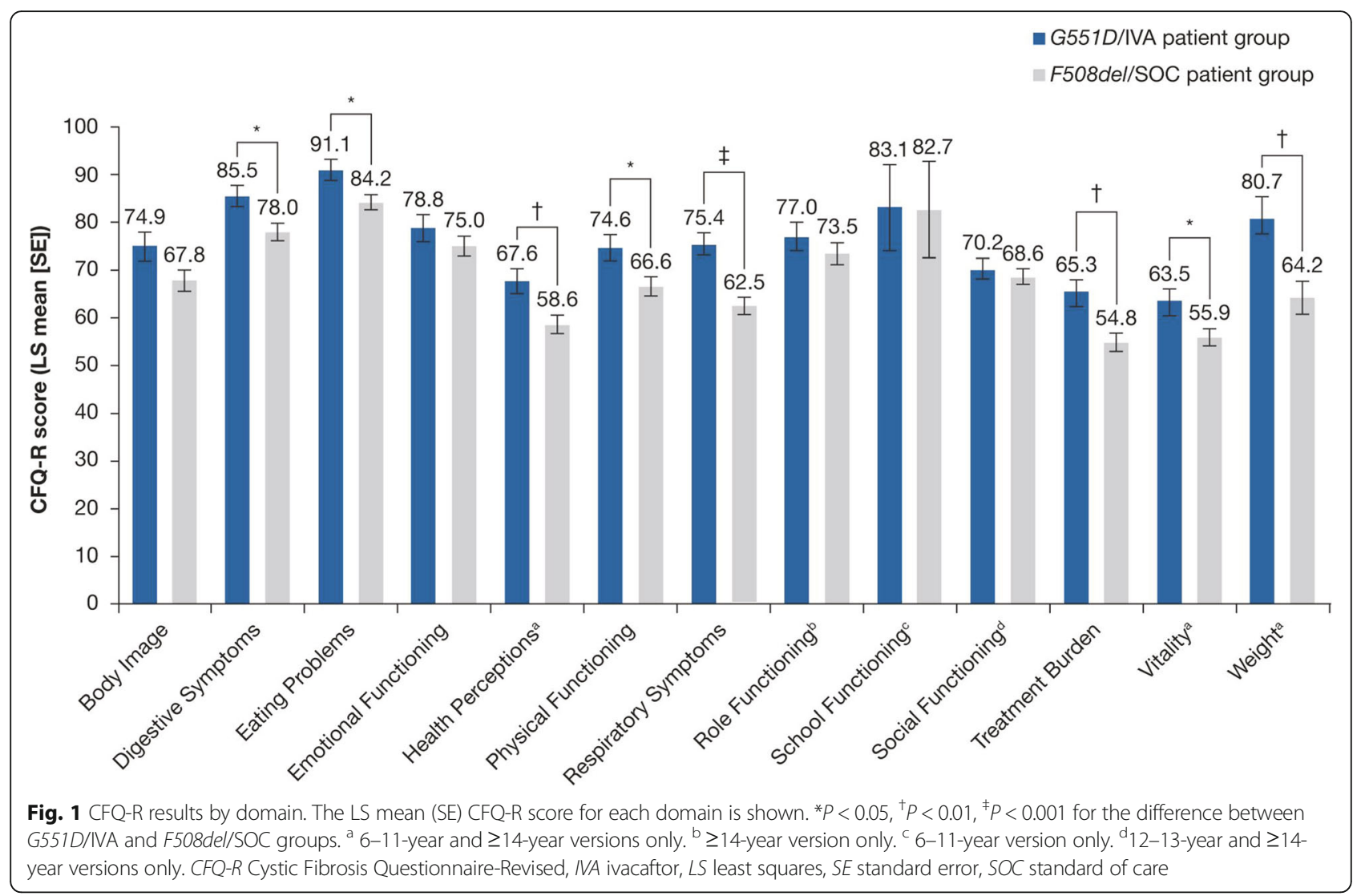

group than in the F508del/SOC group. Although no difference in Self-Care was seen, previous observations in CF have also showed little impact on this scale $[4,10]$. However, Pain/Discomfort and Anxiety/Depression scales have consistently been found to be highly impacted among patients with CF $[4,10]$. These scales may be particularly informative in CF and warrant further study. Depression and anxiety have been reported at higher rates among patients with CF and their caregivers than in the general community [20]. Psychological symptoms in patients with CF have been associated with lower lung function, worse nutritional status, poorer adherence, lower HRQoL, and more frequent hospitalization [21]. Thus, the Cystic Fibrosis Foundation and the European Cystic Fibrosis Society developed a consensus statement for screening and treating depression and anxiety, recommending use of the Patient Health Questionnaire 9- and Generalized Anxiety Disorder 7-item scales for annual screening [21]. In our study, between-group differences were not significant on psychosocial domains of the CFQ-

Table 3 EQ-5D-5L and WPAI results

$$
\text { G551D/IVA patient group }
$$

EQ-5D-5L

Index score (0-1), $n$; LS mean (SE)

VAS score (0-100), $n$; LS mean (SE)

$72 ; 75.7(1.8)$

WPAI

$\begin{array}{ll}\text { School, } n^{\mathrm{a}} & 41 \\ \text { Productivity loss, } n \text {; LS mean (SE) } & 27 ; 24.62(6.69) \\ \text { Daily activities, } n & 70 \\ \text { Activity impairment, LS mean (SE) } & 21.63(2.95)\end{array}$

$137 ; 0.81(0.02)$

$135 ; 70.0(1.4)$

0.0136

53

$32 ; 34.57$ (6.73)

0.3242

135

$28.30(2.19)$ 


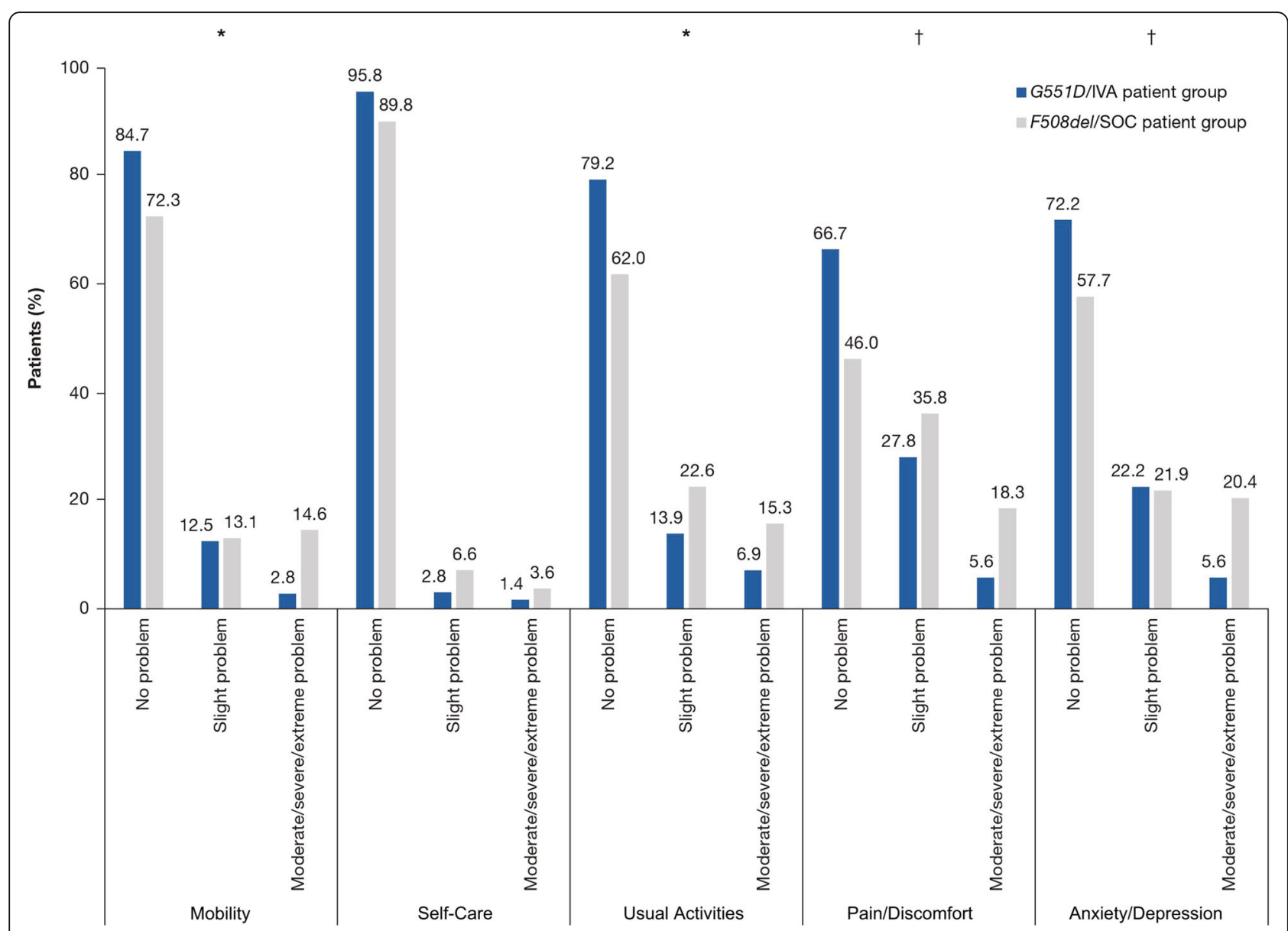

Fig. 2 EQ-5D-5L results by domain. ${ }^{*} P<0.05,{ }^{\dagger} P<0.01$ for the difference in LS means between G551D/IVA and F508del/SOC groups. EQ-5D-5L EuroQol 5-dimensions 5-level questionnaire, IVA ivacaftor, SOC standard of care

$\mathrm{R}$ questionnaire, suggesting that the CFQ- $\mathrm{R}$ questionnaire is less capable of capturing the psychosocial benefits of therapy with CFTR modulators. The role of pain in CF is less well studied, but recent reports indicate that pain is associated with depression, anxiety, lower HRQoL, and increased risk of pulmonary exacerbations and death [22, 23] and could relate to a decrease in the patient's ability to participate in airway clearance activities, or pain could be a potential marker of increased inflammation and/or disease severity. More research is needed to evaluate pain and its effect on HRQoL and other health parameters, as well as to further assess pain management strategies $[22,23]$.

The benefits associated with IVA on the CFQ-R after a mean (SD) exposure of 21.8 (15.1) months were generally consistent with those observed using data from the Phase 3 STRIVE study [17]. Benefits on the Respiratory Symptoms domain were notable; a nearly 13-point difference was found between the G551D/IVA and F508del/SOC groups in adjusted analyses, exceeding the minimum clinically important difference of 4 points for the Respiratory
Symptoms domain of the CFQ-R [24]. Furthermore, these data are consistent with the observational GOAL study, in which real-world patients with G551D mutations treated with IVA for 6 months showed a 7.4-point improvement from baseline in the CFQ-R Respiratory Symptoms domain [18].

Benefits on other CF-specific scales, including Weight, Digestive Symptoms, Eating, and Treatment Burden, were also observed. The between-group difference in Weight score of 16.5 points was particularly notable. Higher scores on general health domains of Physical Functioning, Health Perception, and Vitality were seen, although we did not see higher scores in Social Functioning, as seen in Phase 3 clinical trials [17].

In chronic diseases such as $\mathrm{CF}$, lost productivity can contribute to the overall burden of disease. Additionally, work productivity data are considered in HTAs in several countries, and are considered a relevant and meaningful parameter to evaluate and report because they can further reinforce the value of an intervention from a patient and societal perspective, and can justify social 
funding for interventions that deliver societal value. The WPAI is a PRO tool that can assess productivity losses and general activity impairment but, to our knowledge, has not been previously validated in CF. Our findings show that school productivity loss was significantly lower and activity impairment was numerically lower in the G551D/IVA group than in the F508del/SOC group. While these results are encouraging, they are based on a subgroup of the study population and further research including a larger group of patients is needed.

Several limitations of this cross-sectional, observational study should be noted. The outcomes assessed were compared in patients with different genotypes. There has been debate in the literature regarding whether patients with class III (e.g. G551D) genotypes have disease severity similar to that of patients with class II (e.g. F508del) genotypes. Whereas some studies have shown less severe disease in patients with class III than with class II genotypes, others have found these genotypes to be generally comparable [25-29], although variation in the size of the studies may account for some of the differences reported. The observational nature of the study does not allow for the differences in HRQoL between groups to be directly attributed to IVA, in particular because only one group was exposed to this therapy. For patients in the F508del/SOC group, the exact nature of SOC was not defined, nor were potential differences in the components of what is regarded as SOC in different countries taken into account.

In our sample, we observed differences between groups, such as the G551D/IVA group having a higher proportion of female patients, higher $\mathrm{ppFEV}_{1}$ values, and fewer comorbidities than the F508del/SOC group. These differences may relate to the fact that the patients within the G551D/IVA group were treated with IVA. The significantly lower rate of pancreatic insufficiency seen in the G551D/IVA group, for example, may be associated with IVA treatment, since patient clinical characteristics were collected at the time of the survey when patients were long established on therapy. Furthermore, 3 patients with lung transplants were included in the F508del/SOC group. Ideally, such patients should have been excluded by the study protocol. However, as they constitute only $2.3 \%$ of the F508del/SOC group, and their inclusion likely favors the $F 508 \mathrm{del} / \mathrm{SOC}$ group, we do not envisage that their exclusion would have substantially changed the results. In addition, excluding these patients may favor the results of the G551D/IVA group. Our analysis controlled for differences in patient demographic and clinical characteristics between groups; nevertheless, underlying differences between genotypes that could not be accounted for may still exist. In addition, this was an international multicenter study; therefore, available treatment options and corresponding patient burden may vary across different countries/sites [30]. Furthermore, we saw some imbalances in recruitment across different countries, which differed by group. Although systematic recruitment was attempted, data may reflect patients only in the centers studied, and, as such, these results may not be fully generalizable. As with any cross-sectional, observational study, the data do not capture change over time or treatment effects. However, they do highlight the importance of assessing PROs longitudinally in patients, before and during treatment with a new CFTR modulator therapy. With the growing importance of PROs as a measure of the benefit of therapy from the patients' own perspectives, we would suggest that future longitudinal trials of CFTR modulators in patients with CF should include relevant PROs in their study design. Although resource constraints make routine administration of the CFQ- $R$ impractical in the real-world clinic setting, the simplicity and low costs associated with the EQ-5D-5L confer potential for more widespread use and capture of longitudinal data.

\section{Conclusions}

In summary, patients with CF and a G551D-CFTR mutation on $\geq 1$ allele and receiving IVA in real-world settings reported better generic and disease-specific HRQoL, better school productivity, and better symptomatology than patients with CF who are homozygous for F508delCFTR and receiving SOC (without LUM/IVA) in multiple domains. These data support further longitudinal, multicenter assessments to continue to investigate realworld benefits with IVA in patients with CF and a G551D-CFTR mutation.

\section{Abbreviations}

BMI: Body mass index; CF: Cystic fibrosis; CFQ-R: Cystic Fibrosis Questionnaire-Revised; CFTR: Cystic fibrosis transmembrane conductance regulator; EQ-5D-5L: EuroQol 5-dimensions 5-level questionnaire; HRQOL: Health-related quality of life; HTA: Health technology assessment; IVA: Ivacaftor; LS: Least squares; LUM: Lumacaftor; ppFEV 1 : Percent predicted forced expiratory volume in $1 \mathrm{~s}$; PRO: Patient-reported outcome; SD: Standard deviation; SE: Standard error; SOC: Standard of care; VAS: Visual analog scale; WPAI: Work Productivity and Activity Impairment Questionnaire

\section{Acknowledgements \\ We would like to thank the participating patients and caregivers, as well as the investigators and study coordinators involved in this study. We would also like to thank Emily Hautamaki, Michelle Wood, and Christin Arnold, PhD, for their support with this study. Editorial coordination and support were provided by Gauri Dixit, PhD, and Tejendra Patel, PharmD, who are employees of Vertex Pharmaceuticals Incorporated and may own stock or stock options in that company. Medical writing and editorial support were provided by Stephanie Vadasz, PhD, Michelle Yochum, PhD, and Dena McWain of Ashfield Healthcare Communications and Grace Richmond, PhD, of Excerpta Medica, funded by Vertex Pharmaceuticals Incorporated.}

\section{Authors' contributions}

All authors contributed to the design of the study, provided an interpretation of the data, critically reviewed the manuscript drafts, provided final approval of the manuscript to be submitted, and are accountable for accuracy and integrity of the manuscript. All authors have read and approved the manuscript, and ensure that this is the case. SCB made substantial contributions to the conception and design of the work; the 
acquisition, analysis, and interpretation of data; substantial revisions to the draft; approved the submitted version; agreed both to be personally accountable for their own contributions and to ensure that questions related to the accuracy or integrity of any part of the work, even ones in which they were not personally involved, are appropriately investigated, resolved, and the resolution documented in the literature. JGM made substantial contributions to the conception and design of the work; the acquisition, analysis, and interpretation of data; substantial revisions to the draft; approved the submitted version; agreed both to be personally accountable for their own contributions and to ensure that questions related to the accuracy or integrity of any part of the work, even ones in which they were not personally involved, are appropriately investigated, resolved, and the resolution documented in the literature. GM made substantial contributions to the conception and design of the work; the acquisition, analysis, and interpretation of data; substantial revisions to the draft; approved the submitted version; agreed both to be personally accountable for their own contributions and to ensure that questions related to the accuracy or integrity of any part of the work, even ones in which they were not personally involved, are appropriately investigated, resolved, and the resolution documented in the literature. SM made substantial contributions to the conception and design of the work; the acquisition, analysis, and interpretation of data; substantial revisions to the draft; approved the submitted version; agreed both to be personally accountable for their own contributions and to ensure that questions related to the accuracy or integrity of any part of the work, even ones in which they were not personally involved, are appropriately investigated, resolved, and the resolution documented in the literature. JM made substantial contributions to the conception and design of the work; the acquisition, analysis, and interpretation of data; substantial revisions to the draft; approved the submitted version; agreed both to be personally accountable for their own contributions and to ensure that questions related to the accuracy or integrity of any part of the work, even ones in which they were not personally involved, are appropriately investigated, resolved, and the resolution documented in the literature. MF made substantial contributions to the conception and design of the work; the acquisition, analysis, and interpretation of data; substantial revisions to the draft; approved the submitted version; agreed both to be personally accountable for their own contributions and to ensure that questions related to the accuracy or integrity of any part of the work, even ones in which they were not personally involved, are appropriately investigated, resolved, and the resolution documented in the literature. EDF made substantial contributions to the conception and design of the work; the acquisition, analysis, and interpretation of data; substantial revisions to the draft; approved the submitted version; agreed both to be personally accountable for their own contributions and to ensure that questions related to the accuracy or integrity of any part of the work, even ones in which they were not personally involved, are appropriately investigated, resolved, and the resolution documented in the literature. SN made substantial contributions to the conception and design of the work; the acquisition, analysis, and interpretation of data; substantial revisions to the draft; approved the submitted version; agreed both to be personally accountable for their own contributions and to ensure that questions related to the accuracy or integrity of any part of the work, even ones in which they were not personally involved, are appropriately investigated, resolved, and the resolution documented in the literature. NK made substantial contributions to the conception and design of the work; the acquisition, analysis, and interpretation of data; substantial revisions to the draft; approved the submitted version; agreed both to be personally accountable for their own contributions and to ensure that questions related to the accuracy or integrity of any part of the work, even ones in which they were not personally involved, are appropriately investigated, resolved, and the resolution documented in the literature.

\section{Funding}

This study was funded by Vertex Pharmaceuticals Incorporated. The sponsor supervised study design, study execution, data collection, statistical analyses, data interpretation, and the writing of the report Medical writing and editorial support were provided by Stephanie Vadasz, PhD, Michelle Yochum, PhD, and Dena McWain of Ashfield Healthcare Communications and Grace Richmond, PhD, of Excerpta Medica, funded by Vertex Pharmaceuticals Incorporated.

\section{Availability of data and materials}

The datasets used and/or analyzed during the current study are available from the corresponding author on reasonable request.

\section{Ethics approval and consent to participate}

Central ethics approval of the study protocol was obtained in the UK (national approval via the National Research Ethics Service Committee South Central, reference no. 14/SC/0297) and France (ethics exemption provided by the Comité de Protection des Personnes), while institution-specific ethics approval was obtained at each of the participating institutions from Germany (institutional ethics review committee at Universitätsklinikum Jena [reference no. not available]), and approval by the Ethics Commission of LMU Munich, reference no. 390-14), Australia (local institutional review board at Princess Margaret Hospital, Scientific Advisory Sub-committee, reference no. 2014096/EP, and Human Research Ethics Committee of Prince Charles Hospital, Metro North Hospital and Health Services, reference no. HREC/14/QPCH/ 113), and Ireland (Temple St Children's University Hospital, Ethics Committee, Department of Research, reference no 14.042).

Written informed participant consent was obtained by participants or their guardians. All participants, or their parent or guardian if aged younger than 18 years, provided written informed consent, or assent where appropriate, before enrolment.

\section{Consent for publication}

Not applicable.

\section{Competing interests}

SCB is a site principal investigator, advisory board member, and writing group member; he has received travel support from Vertex Pharmaceuticals Incorporated to attend investigator meetings. He also has been a site principal investigator and received travel support from Galapagos and Flatley to attend investigator meetings and attended advisory board meetings for AbbVie/Galapagos. JGM has participated in clinical trials and has received funding for investigator-initiated research and advisory boards for Vertex Pharmaceuticals Incorporated. GM has received funding for investigator-led research, participated in clinical trials, and attended paid advisory board meetings for Vertex Pharmaceuticals Incorporated. SM is a former advisory board member and has received funding and travel support from Vertex Pharmaceuticals Incorporated. JM has participated in clinical trials and received funding for advisory boards from Vertex Pharmaceuticals Incorporated. MF is a consultant for Vertex Pharmaceuticals Incorporated. EDS is a former employee of Vertex Pharmaceuticals Incorporated and may own stock or stock options in that company. SN is an employee of Decision Resources Group, has provided consulting services for Vertex Pharmaceuticals Incorporated, and has received funding to execute the observational study discussed in this manuscript. NK is an employee of Vertex Pharmaceuticals Incorporated and may own stock or stock options in that company.

\section{Author details}

'Department of Thoracic Medicine, The Prince Charles Hospital and QIMR Berghofer Medical Research Institute, Brisbane, QLD, Australia. Jena University Hospital, Jena, Germany. ${ }^{3}$ Brandenburg Medical School (MHB), University, Brandenburg an der Havel, Germany. ${ }^{4}$ Gartnavel General Hospital, Glasgow, UK. ${ }^{5}$ Royal Brompton Hospital, London, UK. ${ }^{6}$ University Hospital Bordeaux, Bordeaux, France. ${ }^{7}$ AMF Consulting, Los Angeles, CA, USA. ${ }^{8}$ Formerly of Vertex Pharmaceuticals Incorporated, Boston, MA, USA. ${ }^{9}$ Decision Resources Group, Burlington, MA, USA. ${ }^{10}$ Vertex Pharmaceuticals Incorporated, Boston, MA, USA.

\section{Received: 18 March 2019 Accepted: 26 June 2019}

Published online: 13 August 2019

\section{References}

1. Sawicki GS, Rasouliyan L, McMullen AH, Wagener JS, McColley SA, Pasta DJ, et al. Longitudinal assessment of health-related quality of life in an observational cohort of patients with cystic fibrosis. Pediatr Pulmonol. 2011; 46:36-44.

2. Elborn JS. Cystic fibrosis. Lancet. 2016;388:2519-31.

3. Mayer-Hamblett N, Ramsey BW, Kronmal RA. Advancing outcome measures for the new era of drug development in cystic fibrosis. Proc Am Thorac Soc. 2007:4:370-7. 
4. Solem CT, Vera-Llonch M, Liu S, Botteman M, Castiglione B. Impact of pulmonary exacerbations and lung function on generic health-related quality of life in patients with cystic fibrosis. Health Qual Life Outcomes. 2016;14:63.

5. Angelis A, Kanavos P, López-Bastida J, Linertová R, Nicod E, Serrano-Aguilar $P$, et al. Social and economic costs and health-related quality of life in noninstitutionalised patients with cystic fibrosis in the United Kingdom. BMC Health Serv Res. 2015;15:428.

6. Sawicki GS, Goss $\mathrm{CH}$. Tackling the increasing complexity of CF care. Pediatr Pulmonol. 2015;50(suppl 40):S74-9.

7. Modi AC, Quittner AL. Validation of a disease-specific measure of health-related quality of life for children with cystic fibrosis. J Pediatr Psych. 2003;28:535-46.

8. Quittner AL, Buu A, Messer MA, Modi AC, Watrous M. Development and validation of the Cystic Fibrosis Questionnaire in the United States: a healthrelated quality-of-life measure for cystic fibrosis. Chest. 2005;128:2347-54.

9. Rabin R, de Charro F. EQ-5D: a measure of health status from the EuroQol group. Ann Med. 2001;33:337-43.

10. Eidt-Koch D, Mittendorf T, Greiner W. Cross-sectional validity of the EQ-5D-Y as a generic health outcome instrument in children and adolescents with cystic fibrosis in Germany. BMC Pediatr. 2009;9:55.

11. Devlin NJ, Brooks R. EQ-5D and the EuroQol group: past, present and future. Appl Health Econ Health Policy. 2017;15:127-37.

12. Chen H, Blanc PD, Chawla A, Hayden ML, Bleecker ER, Lee JH. Assessing productivity impairment in patients with severe or difficult-to-treat asthma: validation of the Work Productivity Activity Impairment - asthma questionnaire. J Allergy Clin Immunol. 2006;117(suppl(2):S181.

13. Reilly M, Mitchell I, Gooch K, Vo P, Virabhak S, Lorimer M, et al. Preliminary Validation of the Work Productivity Activity Impairment (WPAl) in Caregivers of Children Hospitalized for Respiratory IIIness (WPAl: CHRI) in Germany and Canada Value in Health. 2012;15(7): A650.

14. Ramsey BW, Davies J, McElvaney NG, Tullis E, Bell SC, Dřevínek P, et al. A CFTR potentiator in patients with cystic fibrosis and the G551D mutation. N Engl J Med. 2011;365:1663-72.

15. Davies JC, Wainwright CE, Canny GJ, Chilvers MA, Howenstine MS, Munck A, et al. Efficacy and safety of ivacaftor in patients aged 6 to 11 years with cystic fibrosis with a G551D mutation. Am J Respir Crit Care Med. 2013;187:1219-25.

16. McKone EF, Borowitz D, Dřevínek P, Griese M, Konstan MW, Wainwright C, et al. Long-term safety and efficacy of ivacaftor in patients with cystic fibrosis who have the Gly551Asp-CFTR mutation: a phase 3, open-label extension study (PERSIST). Lancet Respir Med. 2014:2:902-10.

17. Quittner A, Suthoff E, Rendas-Baum R, Bayliss MS, Sermet-Gaudelus I, Castiglione B, et al. Effect of ivacaftor treatment in patients with cystic fibrosis and the G551D-CFTR mutation: patient-reported outcomes in the STRIVE randomized, controlled trial. Health Qual Life Outcomes. 2015:13:93.

18. Rowe SM, Heltshe SL, Gonska T, Donaldson SH, Borowitz D, Gelfond D, et al. Clinical mechanism of the cystic fibrosis transmembrane conductance regulator potentiator ivacaftor in G551D-mediated cystic fibrosis. Am J Respir Crit Care Med. 2014;190:175-84.

19. Nolan CM, Longworth L, Lord J, Canavan JL, Jones SE, Kon SS, et al. The EQ5D-5L health status questionnaire in COPD: validity, responsiveness and minimum important difference. Thorax. 2016;71:493-500.

20. Quittner AL, Goldbeck L, Abbott J, Duff A, Lambrecht P, Solé A, et al. Prevalence of depression and anxiety in patients with cystic fibrosis and parent caregivers: results of the International Depression Epidemiological Study across nine countries. Thorax. 2014;69:1090-7.

21. Quittner AL, Abbott J, Georgiopoulos AM, Goldbeck L, Smith B, Hempstead SE, et al. International Committee on Mental Health in Cystic Fibrosis: Cystic Fibrosis Foundation and European Cystic Fibrosis Society consensus statements for screening and treating depression and anxiety. Thorax. 2016; 71:26-34.

22. Lechtzin N, Allgood S, Hong G, Riekert K, Haythornthwaite JA, Mogayzel P, et al. The association between pain and clinical outcomes in adolescents with cystic fibrosis. J Pain Symptom Manag. 2016;52:681-7.

23. Hayes M, Yaster M, Haythornthwaite JA, Riekert KA, Nelson McMillan K, White $E$, et al. Pain is a common problem affecting clinical outcomes in adults with cystic fibrosis. Chest. 2011;140:1598-603.

24. Quittner AL, Modi AC, Wainwright C, Otto K, Kirihara J, Montgomery AB. Determination of the minimal clinically important difference scores for the Cystic Fibrosis Questionnaire-Revised Respiratory Symptom Scale in two populations of patients with cystic fibrosis and chronic Pseudomonas aeruginosa airway infection. Chest. 2009;135:1610-8.
25. Comer DM, Ennis M, McDowell C, Beattie D, Rendall J, Hall V, et al. Clinical phenotype of cystic fibrosis patients with the G551D mutation. QJM. 2009; 102:793-8.

26. Dupuis A, Keenan K, Ooi CY, Dorfman R, Sontag MK, Naehrlich L, et al. Prevalence of meconium ileus marks the severity of mutations of the cystic fibrosis transmembrane conductance regulator (CFTR) gene. Genet Med. 2016;18:333-40.

27. Hamosh A, King TM, Rosenstein BJ, Corey M, Levison H, Durie P, et al. Cystic fibrosis patients bearing both the common missense mutation Gly >Asp at codon 551 and the $\triangle$ F508 mutation are clinically indistinguishable from $\triangle F 508$ homozygotes, except for decreased risk of meconium ileus. Am J Hum Genet. 1992;51:245-50.

28. McKone EF, Emerson SS, Edwards KL, Aitken ML. Effect of genotype on phenotype and mortality in cystic fibrosis: a retrospective cohort study. Lancet. 2003;361:1671-6.

29. Sawicki GS, McKone EF, Millar SJ, Pasta DJ, Konstan MW, Lubarsky B, et al. Patients with cystic fibrosis and a G551D or homozygous F508del mutation: similar lung function decline. Am J Respir Crit Care Med. 2017;195:1673-6.

30. McCormick J, Mehta G, Olesen HV, Viviani L, Macek M Jr, Mehta A, et al. Comparative demographics of the European cystic fibrosis population: a cross-sectional database analysis. Lancet. 2010;375:1007-13.

\section{Publisher's Note}

Springer Nature remains neutral with regard to jurisdictional claims in published maps and institutional affiliations.
Ready to submit your research? Choose BMC and benefit from:

- fast, convenient online submission

- thorough peer review by experienced researchers in your field

- rapid publication on acceptance

- support for research data, including large and complex data types

- gold Open Access which fosters wider collaboration and increased citations

- maximum visibility for your research: over $100 \mathrm{M}$ website views per year

At BMC, research is always in progress.

Learn more biomedcentral.com/submissions 